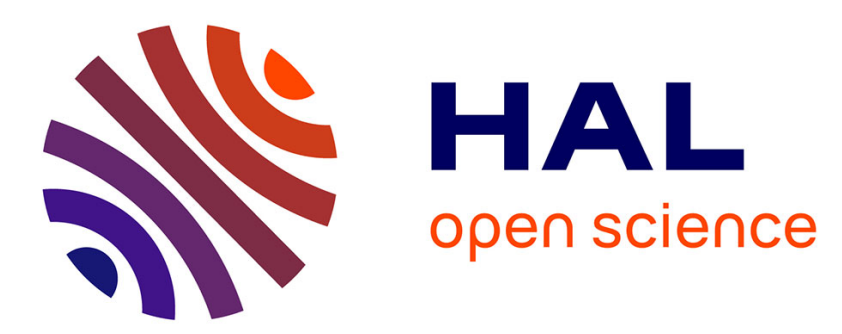

\title{
Analyse des propriétés de transport électrique dans le silicium sur isolant - Utilisation du pouvoir thermoélectrique
}

\author{
G. Ghibaudo, G. Kamarinos
}

\section{- To cite this version:}

G. Ghibaudo, G. Kamarinos. Analyse des propriétés de transport électrique dans le silicium sur isolant - Utilisation du pouvoir thermoélectrique. Revue de Physique Appliquée, 1982, 17 (3), pp.133-143. 10.1051/rphysap:01982001703013300 . jpa-00244981

\section{HAL Id: jpa-00244981 https://hal.science/jpa-00244981}

Submitted on 1 Jan 1982

HAL is a multi-disciplinary open access archive for the deposit and dissemination of scientific research documents, whether they are published or not. The documents may come from teaching and research institutions in France or abroad, or from public or private research centers.
L'archive ouverte pluridisciplinaire HAL, est destinée au dépôt et à la diffusion de documents scientifiques de niveau recherche, publiés ou non, émanant des établissements d'enseignement et de recherche français ou étrangers, des laboratoires publics ou privés. 


\title{
Analyse des propriétés de transport électrique dans le silicium sur isolant - Utilisation du pouvoir thermoélectrique
}

\author{
G. Ghibaudo et G. Kamarinos \\ Laboratoire de Physique des Composants à Semiconducteurs $\left(^{*}\right)$ \\ ENSER, 23, rue des Martyrs, 38031 Grenoble Cedex, France
}

(Reçu le 5 octobre 1981, révisé le 23 novembre 1981, accepté le 8 décembre 1981)

\begin{abstract}
Résumé. - L'utilisation du pouvoir thermoélectrique (PTE) pour l'étude des propriétés de transport des semiconducteurs inhomogènes est proposée lors de ce travail. Plus particulièrement le Silicium sur Saphir (SSI) de type $\mathbf{N}$ (couche mince de silicium, inhomogène dans le sens de sa croissance épitaxiale) sert de matériau de référence et de test. Une étude théorique d'abord, de la conductivité électrique, de l'effet Hall et du PTE de tels échantillons est présentée; les variations des mobilités et des densités de porteurs avec la température dans les différentes parties de l'échantillon inhomogène sont prises en considération. Un modèle théorique numérique pour des matériaux pouvant être considérés comme étant constitués de deux couches à propriétés différentes (matériaux bicouches) est ensuite établi. A l'aide de ce modèle théorique une comparaison détaillée entre l'effet Hall et le PTE relativement à leur pertinence pour l'étude d'un matériau bicouche est effectuée. Nous montrons ainsi que les mesures du PTE sont beaucoup plus sensibles aux inhomogénéités que les mesures d'effet Hall. Dans le cas du SSI l'étude de la sensibilité du PTE $(S)$ et de la conductivité électrique $(\sigma)$ en fonction des variations des paramètres globaux de transport montre que les mesures conjuguées de $\sigma$ et de $S$ peuvent s'avérer suffisantes pour une caractérisation efficace et satisfaisante du matériau dont un des principaux problèmes est son inhomogénéité épitaxiale. Des mesures effectuées sur des échantillons de SSI dopés au phosphore sont ensuite présentées et analysées. Ces échantillons d'une épaisseur de $0,7 \mu \mathrm{m}$, sont étudiés dans une gamme de température de $77 \mathrm{~K}$ à $360 \mathrm{~K}$. A partir des variations de leur conductivité électrique et de leur PTE nous déduisons leurs paramètres de transport (densités et mobilités des porteurs ainsi que leurs variations). Les échantillons étudiés correspondent bien au modèle théorique bicouche que nous avons élaboré. Ils présentent une structure $\mathrm{N}-\mathrm{N}^{+}$. La couche dégénérée $\mathrm{N}^{+}$, d'une épaisseur déduite de $150 \AA$, constitue la région de transition entre le substrat isolant et la partie principale monocristalline du film. Elle pourrait être soit une région surdopée soit une couche d'accumulation forte.
\end{abstract}

\begin{abstract}
The use of the thermoelectric power (TEP) for the study of the transport properties of inhomogeneous semiconductors is proposed. Particularly, in this work, the Silicon On Sapphire (SOS) of N type is considered as a reference and as a test material; indeed the SOS films are inhomogeneous in the direction of their epitaxial growth. In a first time a theoretical study of the electrical conductivity, the Hall effect and the TEP of such samples is presented ; the variations of the mobilities and the densities of free carriers with temperature in the different parts of the inhomogeneous samples are taken into account. A numerical theoretical model concerning semiconductors which can be considered as two (different) layers materials is established. Using the above theoretical model a detailed comparison between the Hall effect and the TEP is performed. We show that the measurements of TEP are much more sensitive to transport inhomogeneities than Hall effect measurements. In the case of SOS, the study of the sensitivity of the TEP $(S)$ and the electrical conductivity $(\sigma) v s$. the variations of the transport parameters, show that the combined measurements of $\sigma$ and $S$ can be sufficient for a satisfactory and efficient characterization of this material the main problem of which is, precisely, its epitaxial inhomogeneity. Experimental results performed on phosphorus doped SOS films are presented and analysed. The thickness of the films is about $0.7 \mu \mathrm{m}$; they are studied between $77 \mathrm{~K}$ and $360 \mathrm{~K}$. The analysis of $(\sigma, T)$ and $(S, T)$ characteristics leads to the transport parameters as well as to the law of their variations. We deduce that the SOS samples are very satisfactorily represented by the main $\mathrm{N}$ type part and a degenerate, $\mathrm{N}^{+}$type, thin layer ; the thickness of the $\mathrm{N}^{+}$type transition $\left(\mathrm{Si}_{-}-\mathrm{Al}_{2} \mathrm{O}_{3}\right)$ layer is estimated on the order of $150 \AA$. The transition layer is either a overdoped and highly disturbed region or a strongly accumulated layer.
\end{abstract}


1. Introduction. - La microélectronique actuelle nécessite de plus en plus l'emploi de nouveaux matériaux sous forme de couches minces semiconductrices. De telles couches - homoépitaxiées ou hétéroépitaxiées - posent alors le problème de la caractérisation des propriétés de transport, dont les valeurs ne sont plus constantes selon l'axe de croissance de la couche. Tel est le cas, en particulier du Silicium sur Isolant (SSI ou SOS : Silicon On Sapphire), dont l'utilité en VLSI, est bien connue [1,2].

Précédemment, différentes études ont montré l'existence d'un gradient des propriétés électriques selon la direction d'épitaxie : l'effet magnétodiode a permis de mettre en évidence un gradient de durée de vie des porteurs [3] comme cela a été entrevu à l'aide de méthodes de mesures indirectes [4] et [5]. Des profils de mobilités et de concentrations des porteurs ont pu être établis grâce à des mesures d'effet $\mathrm{Hall}$ et de magnétorésistance sur des structures MOSFET à déplétion $[5,6,7,8$ et 32]. Signalons que l'étude du transistor MOS métal- $\mathrm{Al}_{2} \mathrm{O}_{3}-\mathrm{Si}$ a confirmé la faible mobilité des porteurs au voisinage de l'interface $\mathrm{Si}-\mathrm{Al}_{2} \mathrm{O}_{3}$ [9]. Curieusement, dans la littérature, si l'étude des propriétés de transport est très largement représentée par la conductivité et l'effet Hall, le pouvoir thermoélectrique (PTE) est en général absent. Ceci n'est pas surprenant quand on connaît les difficultés expérimentales inhérentes aux mesures du PTE.

Dans cet article, nous présentons une étude théorique détaillée des propriétés de transport (conductivité, effet Hall, PTE) dans un matériau semiconducteur inhomogène, et montrons que, dans ce cas, l'intérêt du PTE non seulement n'est plus secondaire par rapport à l'effet Hall, mais qu'au contraire celui-ci s'avère plus sensible à la présence d'une inhomogénéité transversale. Nous appliquons ensuite, notre analyse théorique aux résultats expérimentaux du PTE et de la conductivité obtenus sur des échantillons de SSI de type $\mathbf{N}$. Nous en déduisons une image globale de l'inhomogénéité du film selon l'axe épitaxial et en particulier nous confirmons le rôle déterminant joué par la couche de transition à l'interface $\mathrm{Si}_{\mathrm{i}} \mathrm{Al}_{2} \mathrm{O}_{3}$, dans le transport électrique à basse température.

2. Analyse théorique. - 2.1 GÉNÉRALITÉs. - Avant d'aborder le détail des calculs des propriétés de transport dans le cas d'un matériau transversalement inhomogène, nous devons formuler quelques remarques générales concernant le formalisme mathématique employé. Dans ce qui suit, nous allons considérer que l'inhomogénéité transversale est suffisamment abrupte pour donner lieu à l'apparition de deux couches fictives dans l'épaisseur du film, dont les propriétés électriques sont très différentes. $\mathrm{Ce}$ « découpage » en deux régions bien distinctes, de l'échantillon pourrait paraître trop artificiel, cependant notre approche simplificatrice, à cet égard, considère seulement des matériaux fortement inhomogènes dans lesquels seuls des effets d'inhomogénéités qualitativement très différents, sont pris en compte. Plus

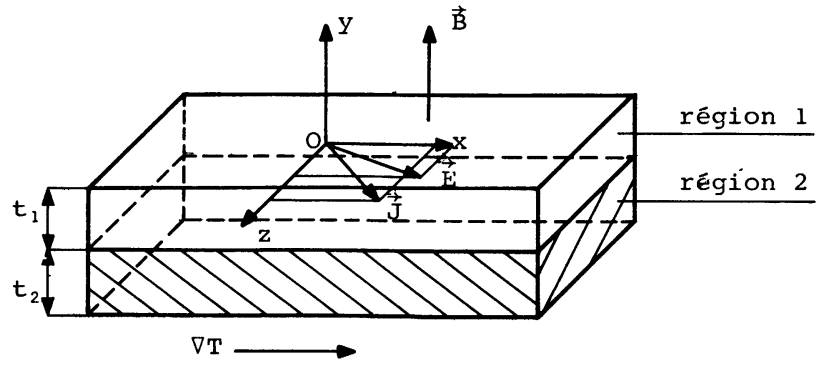

Fig. 1. - Echantillon bicouche (régions 1 et 2) dans lequel sont définis pour chacune des couches :

$\mathbf{E}\left(E_{x}, 0, E_{z}\right)$ champ électrique, $\mathbf{B}\left(0, B_{y}, 0\right)$ champ magnétique, $\mathbf{j}\left(j_{x}, 0, j_{z}\right)$ densité de courant,

$\nabla T\left((\nabla T)_{x}, 0,0\right)$ gradient de température,

$t_{1}$ épaisseur de la région 1 ,

$t_{2}$ épaisseur de la région 2 .

[Two-layers sample (regions 1 and 2) in which are defined :

$\mathbf{E}\left(E_{x}, 0, E_{z}\right)$ electrical field,

$\mathbf{B}\left(0, B_{y}, 0\right)$ magnetic field,

i $\left(j_{x}, 0, j_{z}\right)$ current density,

$\nabla T\left((\nabla T)_{x}, 0,0\right)$ temperature gradient,

$t_{1}$ thickness of the region 1 ,

$t_{2}$ thickness of the region 2.]

particulièrement en ce qui concerne le SSI, on a, par ailleurs $[5,7,8]$, la conviction qu'effectivement, il est constitué grossièrement de deux régions : la couche de transition à l'interface $\mathrm{Si}-\mathrm{Al}_{2} \mathrm{O}_{3}$ de l'ordre de quelques centaines d'angströms et la couche supérieure du film. Il est néanmoins évident qu'une analyse plus fine des inhomogénéités transversales nécessiterait un formalisme mathématique différentiel plus complexe [11], dont les principes physiques d'établissement sont identiques à ceux que nous allons mentionner dans les paragraphes suivants. En ce qui nous concerne, l'utilité d'un tel formalisme n'était pas justifié dans la mesure où le SSI étudié, présentait un caractère inhomogène suffisant, se prêtant à une analyse simplifiée. Dans cette partie, nous développerons donc les calculs donnant la conductivité $\sigma$, la mobilité de Hall $\mu_{\mathrm{H}}$, et le PTE $S$, dans le cas d'un matériau bicouche, constitué de deux régions distinctes (cf. Fig. 1). Chacune des deux régions, supposées homogènes et isotropes, présente ses propres paramètres de transport : $\sigma_{1}, \mu_{\mathrm{H} 1}, S_{1}$ pour la couche 1 et $\sigma_{2}, \mu_{\mathrm{H} 2}, S_{2}$ pour la couche 2 . Nous déduirons, théoriquement, les expressions donnant les paramètres globaux en fonction des paramètres de transport élémentaires de chacune des couches. Ensuite nous appliquerons ce formalisme, au cas d'un matériau constitué d'une couche principale de silicium $\mathrm{N}$ et d'une mince couche de silicium très dopé $\mathrm{N}^{+}$. Cet échantillon hypothétique servira de référence lors de l'évaluation. numérique des paramètres globaux de transport $\sigma, \mu_{\mathrm{H}}$ et $S$ d'un matériau réel transversalement inhomogène. Il constituera ensuite un élément de comparaison utile à l'interprétation des propriétés 
de transport du SSI que nous présenterons dans la troisième partie.

2.2 ConduCtivité ÉLECTRIQUe. - La conductivité électrique $\sigma$, relie la densité de courant $\mathbf{j}$ au champ électrique $\mathbf{E}$ selon la relation d'Ohm :

$$
\mathbf{j}=\sigma \mathbf{E} .
$$

Dans le cas de notre échantillon bicouche (transversalement inhomogène), le champ électrique et la densité de courant sont supposés colinéaires à l'axe $\mathrm{O} X$ (cf. Fig. 1) : $\mathbf{E}\left(E_{x}, 0,0\right)$ et $\mathbf{j}\left(j_{x}, 0,0\right)$ (ici $\left.j_{z} \equiv 0\right)$. De la conservation du courant total $(\operatorname{div} \mathbf{j}=0)$ et de la composante parallèle du champ électrique (découlant de $\operatorname{rot} \mathbf{E}=0$ ), dans le plan $(\mathrm{O} x z)$, nous déduisons les équations suivantes :

$$
\begin{aligned}
\left(t_{1}+t_{2}\right) j_{x} & =t_{1} j_{1 x}+t_{2} j_{2 x} \\
j_{1 x} & =\sigma_{1} E_{1 x} \\
j_{2 x} & =\sigma_{2} E_{2 x} \\
E_{1 x} & =E_{2 x}=E_{x}
\end{aligned}
$$

où $j_{\mathrm{ix}}$ est la densité de courant dans la région $\mathrm{i}, E_{\mathrm{i} x}$ le champ électrique dans la région $\mathrm{i}, t_{\mathrm{i}}$ l'épaisseur de chaque région selon l'axe oy. La conductivité équivalente de l'échantillon $\sigma$, définie par $j_{x}=\sigma E_{x}$, s'écrit donc à l'aide des équations (1) à (5) :

$$
\sigma=\frac{\lambda}{1+\lambda} \sigma_{1}+\frac{1}{1+\lambda} \sigma_{2}
$$

où $\lambda=t_{1} / t_{2}$ est le facteur géométrique exprimant l'importance relative de la région 1 sur la région 2 . Rappelons que les conductivités de chacune des couches i sont reliées aux mobilités $\mu_{\mathrm{i}}$ et aux densités de porteurs $n_{\mathrm{i}}$ par les relations usuelles : $\sigma_{\mathrm{i}}=|q| \mu_{\mathrm{i}} n_{\mathrm{i}}$ où $q$ est la charge de l'électron.

2.3 EfFet Hall. - Nous calculerons la mobilité de Hall $\mu_{\mathrm{H}}$ et la constante de Hall $R_{\mathrm{H}}$ à partir de l'équation de transport de Boltzmann $[10,12]$ dans l'approximation des champs magnétiques faibles, ce qui nous conduira à négliger la magnétorésistance. Le champ électrique total dans le plan oxz étant conservé, on peut écrire pour notre échantillon (cf. Fig. 1) :

$$
\mathbf{E}=\frac{1}{\sigma_{1}} \mathbf{j}_{1}+\frac{\mu_{\mathrm{H} 1}}{\sigma_{1}}\left(\mathbf{B} \times \mathbf{j}_{1}\right)=\frac{1}{\sigma_{2}} \mathbf{j}_{2}+\frac{\mu_{\mathrm{H} 2}}{\sigma_{2}}\left(\mathbf{B} \times \mathbf{j}_{2}\right)
$$

où $\mathbf{B}$ est le champ magnétique appliqué. D'autre part, de la conservation du courant selon ox et $o z$, résultent l'équation (2) et la relation suivante :

$$
t_{1} j_{1 z}+t_{2} j_{2 z}=0 \text {. }
$$

Appliquant un champ magnétique dans le sens de l'inhomogénéité $\mathbf{B}\left(0, B_{y}, 0\right)$ et projetant l'équation (7) sur les axes, on obtient :

$$
\begin{aligned}
& \frac{1}{\sigma_{1}} j_{1 x}+\frac{\mu_{\mathrm{H} 1}}{\sigma_{1}} B_{y} j_{1 z}=\frac{1}{\sigma_{2}} j_{2 x}+\frac{\mu_{\mathrm{H} 2}}{\sigma_{2}} B_{y} j_{2 z} \\
& \frac{1}{\sigma_{1}} j_{1 z}+\frac{\mu_{\mathrm{H} 1}}{\sigma_{1}} B_{y} j_{1 x}=\frac{1}{\sigma_{2}} j_{2 z}+\frac{\mu_{\mathrm{H} 2}}{\sigma_{2}} B_{y} j_{2 x} .
\end{aligned}
$$

La relation de Hall définissant la mobilité de Hall s'écrit alors

$$
E_{\mathrm{Hz}}=\frac{\mu_{\mathrm{H}}}{\sigma} j_{x} \quad\left(\text { où } \sigma=\frac{\lambda \sigma_{1}+\sigma_{2}}{\lambda+1} \text { cf. } 2.2\right)
$$

où $E_{\mathrm{Hz}}$ est le champ électrique de Hall apparaissant dans la direction $o z$. En remarquant qu'à faible champ magnétique $(\mu B \ll 1)$ on a $j_{1 x}=\sigma_{1} E_{1 x}$ et $j_{2 x}=\sigma_{2} E_{2 x}$; il vient à l'aide des équations (2) et (8) à $(10)$ :

$$
\mu_{\mathrm{H}}=\frac{\lambda \sigma_{1} \mu_{\mathrm{H} 1}+\sigma_{2} \mu_{\mathrm{H} 2}}{\lambda \sigma_{1}+\sigma_{2}} \quad\left(\text { où } \lambda=\frac{t_{1}}{t_{2}}\right)
$$

d'où également, sachant que $R_{\mathrm{H}}=\mu_{\mathrm{H}} / \sigma$, on déduit le coefficient de Hall $R_{\mathrm{H}}$ :

$$
R_{\mathrm{H}}=\frac{\lambda \sigma_{1}^{2} R_{\mathrm{H} 1}+\sigma_{2}^{2} R_{\mathrm{H} 2}}{\left(\lambda \sigma_{1}+\sigma_{2}\right)^{2}}
$$

où les $R_{\mathrm{Hi}}$ sont les constantes de Hall dans chaque couche $\mathrm{i}$.

2.4 Pouvoir thermó́lectrique. - Calculons le PTE $S$ de notre échantillon de la figure 1 soumis à un gradient de température $\nabla T\left((\nabla T)_{x}, 0,0\right)$ selon l'axe Ox, en l'absence de toute autre perturbation $(\mathbf{j} \equiv 0$, $\mathbf{B} \equiv 0)$. D'après $[10,12,15]$ le champ électrique total est alors la somme du champ Seebeck $S \nabla T$ et du champ de conduction $\mathbf{j} / \sigma$, donc :

$$
\mathbf{E}=\frac{\mathbf{j}}{\sigma}+S \nabla T .
$$

De plus le courant total selon Ox étant nul (condition de circuit ouvert), il en résulte :

$$
t_{1} j_{1 x}+t_{2} j_{2 x}=0 \text {. }
$$

Le champ électrique Seebeck $\mathbf{E}_{\mathrm{s}}$ n'est alors autre que la valeur commune du champ électrique dans les deux régions 1 et 2 , soit :

$$
\left(E_{\mathrm{s}}\right)_{x}=\frac{j_{1 x}}{\sigma_{1}}+S_{1}(\nabla T)_{x}=\frac{j_{2 x}}{\sigma_{2}}+S_{2}(\nabla T)_{x} .
$$

On obtient alors pour l'expression du PTE global d'un échantillon bicouche à l'aide des relations (6), (14) et (15) :

$$
S=\frac{\lambda \sigma_{1} S_{1}+\sigma_{2} S_{2}}{\lambda \sigma_{1}+\sigma_{2}}
$$

2.5 Comparaison efFet Hall-PTE POUR UN ÉCHANTILLON BICOUCHE SiN-N ${ }^{+}$. - Dans ce para- 
graphe nous allons appliquer à un matériau semiconducteur inhomogène constitué de deux couches de silicium $\mathrm{N}$, les résultats obtenus aux paragraphes 2.3 et 2.4 , afin d'analyser l'influence d'une mince couche surdopée $\mathrm{N}^{+}$sur les propriétés électriques globales en fonction de la température dont nous limiterons les variations entre $60 \mathrm{~K}$ et $360 \mathrm{~K}$, en raison des exigences expérimentales ( $3 \mathrm{e}$ partie). A notre échantillon de référence (cf. Fig. 1), nous affecterons à la région 1 les propriétés du silicium massif de type $\mathrm{N}$ et à la région 2 celles du silicium dégénéré de type $\mathrm{N}^{+}$. Nous calculerons, ensuite, numériquement l'évolution de la mobilité de Hall $\mu_{\mathrm{H}}$ et du PTE $S$ en fonction de la température. Pour cela il nous faut maintenant préciser quelques hypothèses concernant d'une part les lois de mobilité et, d'autre part, les densités de porteurs. Nous déduirons ainsi les paramètres de transport élémentaires (cf. 2.2, $2.3,2.4)$ de chacune des couches.

2.5.1 Expressions des mobilités. - Les lois de mobilités en fonction de la température $T$, que nous allons définir sont inspirées par l'analyse de nombreuses données expérimentales relatives au $\mathrm{Si}[10,14$, 29] dont la compatibilité avec les expressions théoriques est sensiblement vérifiée $[12,13,14]$. Dans la région 1 , le matériau semiconducteur dopé normalement $\left(N_{\mathrm{d}}<10^{18} / \mathrm{cm}^{3}\right)$, admet un mécanisme de diffusion des porteurs mixte. A haute température $(250 \mathrm{~K}<T<350 \mathrm{~K})$ la diffusion est contrôlée par les vibrations du réseau (phonons acoustiques) et donne lieu théoriquement à des variations de mobilité en $T^{-3 / 2}$. A basse température $(60 \mathrm{~K}<T<200 \mathrm{~K})$ la diffusion est contrôlée par les impuretés ionisées ou les défauts ponctuels chargés (diffusion coulombienne) $[12,13]$ et conduit à des lois de mobilité en $T^{3 / 2}$. La mobilité globale, en présence de plusieurs mécanismes de diffusion est alors obtenue à partir de la règle de Mathiessen [12, 13] (addition inverse des mobilités); il vient pour l'expression de la mobilité dans la région 1 :

$$
\mu_{1}=2 \mu_{\mathrm{M}}\left(\left(\frac{T}{T_{\mathrm{M}}}\right)^{1,5}+\left(\frac{T_{\mathrm{M}}}{T}\right)^{1,5}\right)^{-1}
$$

où $\mu_{\mathrm{M}}$ est la valeur maximum de la mobilité réalisée pour la température $T_{\mathrm{M}}$. Dans la région 2, le matériau est dégénéré et par conséquent admet, en première approximation, un mécanisme de diffusion quasi indépendant de la température $[10,13,14]$. De plus, les données du SSI $[5,8,9]$ et les données du Si $\left(\mathrm{N}^{+}\right)$ permettent d'affecter à la mobilité dans la région 2 une valeur moyenne $\mu_{2}$ de l'ordre de quelques dizaines de $\mathrm{cm}^{2} /$ V.s. Par la suite, pour alléger le modèle numérique, nous confondrons les mobilités de dérive avec les mobilités de Hall. Cela revient à dire que, le coefficient de proportionnalité entre ces deux mobilités, fonction du mécanisme de diffusion, sera posé égal à $1[10,12,28]$. Cette approximation, vu les valeurs des paramètres de diffusion utilisées nous conduira à une erreur maximale sur les mobilités, estimée à $30 \%$ environ. Nous poserons donc par la suite $\mu_{\mathrm{Hi}} \simeq \mu_{\mathrm{i}}$.

Avant d'en terminer avec cette partie consacrée aux mobilités, nous voudrions formuler quelques remarques, quant aux mécanismes de diffusion : il est clair que les lois de mobilités que nous avons considérées pour la simulation numérique, ne sont sans doute pas les seules envisageables pour tenir compte des effets' des imperfections du matériau. En particulier, d'autres mécanismes de diffusion tels que, par exemple, diffusion par impuretés neutres, par défauts cristallographiques, etc... sont tout à fait possibles $[5,7,8]$. Cependant, devant l'incertitude des expressions théoriques, d'une part, et le manque de données à cet égard, d'autre part, il nous est apparu plus prudent de conserver les mécanismes de diffusion prépondérants dont l'ajustement aux courbes expérimentales est le plus communément admis [7, 8, 10, 12, 13] en ce qui concerne le Si et le SSI.

2.5.2 Expressions des niveaux de Fermi. - Pour le calcul des densités de porteurs $n_{1}$ et $n_{2}$ des régions 1 et 2 de notre échantillon de référence (cf. Fig. 1), il nous est nécessaire de définir et de calculer en fonction de la température, les niveaux de Fermi $E_{\mathrm{F}_{1}}$ et $E_{\mathrm{F}_{2}}$ repérés à partir de la bande de conduction. Le signe du niveau de Fermi, pour un matériau $\mathrm{N}$, étant ainsi pris négatif s'il est à l'intérieur de la bande interdite. Dans la région 1 , la statistique du matériau non dégénéré prévaut. Elle conduit, grâce à l'équation de neutralité, à l'expression du niveau de Fermi valable jusqu'à la température d'exhaustion (450 K pour le Si) $[10,12]$ :

$$
E_{\mathrm{F}_{1}}=E_{\mathrm{d}}+k T \log \left(\frac{1}{4}\left(\sqrt{1+\frac{8 N_{\mathrm{d}}}{N_{\mathrm{c}}} \exp \left(-\frac{E_{\mathrm{d}}}{k T}\right)}-1\right)\right) \text {, }
$$

où :

$E_{\mathrm{d}}$ est l'énergie d'ionisation des donneurs située dans le gap et reférée à la bande de conduction $\left(E_{d}<0\right)$,

$k$ est la constante de Boltzmann,

$N_{\mathrm{d}}$ la densité de donneurs,

$N_{\mathrm{c}}$ est la densité d'états ramenés au bas de la bande de conduction, pour le Si elle s'exprime par $[10,12]$ :

$$
N_{\mathrm{c}}=2,7 \times 10^{19}\left(\frac{T}{300}\right)^{1,5} / \mathrm{cm}^{3} .
$$

La densité de porteurs $n_{1}$ se déduit alors aisément pour chaque température par la relation bien connue :

$$
n_{1}=N_{\mathrm{c}} \exp \left(\frac{E_{\mathrm{F}_{1}}}{k T}\right) \text {. }
$$

Pour la région 2, la statistique du semiconducteur dégénéré est nécessaire. Dans ce cas, le niveau de Fermi $E_{\mathrm{F}_{2}}$ passe au-dessus de la bande de conduction et il est donné par l'expression relative aux métaux [10, 12] :

$$
E_{\mathrm{F}_{2}}=\frac{\hbar^{2}}{2 m^{*}}\left(3 \pi^{2} n_{2}\right)^{2 / 3}
$$


où $\hbar$ est la constante réduite de Planck $(h / 2 \pi), m^{*}$ la masse effective de la densité d'états (pour le $\mathrm{Si}$, $m^{*} \simeq m_{0} ; m_{0}$ étant la masse de l'électron libre). Dans ces conditions, le niveau de Fermi $E_{\mathrm{F}_{2}}$ ainsi que la densité de porteurs $n_{2}$, sont pratiquement indépendants de la température (cas métallique).

Par la suite, nous utiliserons pour la simulation numérique, des valeurs de $n_{2}$ de l'ordre de $10^{19}$ à $10^{20} / \mathrm{cm}^{3}$, dont on sait qu'elles assurent la dégénérescence dans le cas du silicium $\mathrm{N}^{+}[10,14,29]$.

2.5.3 Expression des pouvoirs thermoélectriques. Le pouvoir thermoélectrique d'une couche homogène semiconductrice, dans laquelle la notion de bande de conduction est applicable, est donné par des expressions déduites de l'équation de transport de Boltzmann $[10,12,13]$. Avant de donner le détail de ces expressions, rappelons quelques propriétés générales du PTE : celui-ci est une grandeur intensive, c'est-àdire indépendante des dimensions de l'échantillon; cette propriété est très intéressante car la mesure du PTE ne nécessite pas des échantillons de taille et de forme géométrique bien déterminées. De plus, c'est une grandeur algébrique dont le signe dépend de celui des porteurs majoritaires $(S<0$ par convention pour des électrons); enfin il est linéairement dépendant du mécanisme de diffusion des porteurs, et peut, par conséquent, donner lieu à une analyse fine des processus de collisions [15, 16, 27]. En revanche, comme nous l'avons vu au paragraphe 2.4 , dans le cas d'un matériau inhomogène, le PTE dépend d'une manière compliquée des effets et paramètres précédents, et il ne peut plus être analysé d'une façon simple au moyen des formules classiques valables pour un matériau isotrope et homogène.

Dans la couche 1, homogène et non dégénérée, le PTE s'obtient par l'expression classique $[12,15,27]$ :

$$
S_{1}=\frac{k}{q}\left(-\frac{E_{\mathrm{F}_{1}}}{k T}+\frac{\Delta E_{\mathrm{C}}}{k T}\right)
$$

où $k, q, T$ ont été définis précédemment, $E_{\mathrm{F}_{1}}$ est le niveau de Fermi dans la région 1 donné par (18), $\Delta E_{\mathrm{C}}$ est l'énergie cinétique moyenne transportée par l'électron dans la bande de conduction et dépend exclusivement du mécanisme de diffusion $\left(\Delta E_{\mathrm{C}} / k T=2\right.$ pour une diffusion par les phonons acoustiques; $\Delta E_{\mathrm{C}} / k T=4$ pour une diffusion par les impuretés ionisées $[12,15,16])$.

Dans la couche 2, homogène et dégénérée, le PTE s'obtient par l'expression classique des semiconducteurs dégénérés $[12,15,16]$ :

$$
S_{2}=\frac{\pi^{2}}{3} \frac{k}{q} \frac{k T}{E_{\mathrm{F}_{2}}}\left(\alpha+\frac{3}{2}\right)
$$

où $E_{\mathrm{F}_{2}}$ est le niveau de Fermi dans la région 2 donné par la relation (21), $\alpha$ est le coefficient de dépendance en énergie $E$ du temps de relaxation $\tau$ défini par : $\tau \sim E^{\alpha}$.

Dans le cas du Si- $\mathrm{N}^{+}$, qui nous intéresse, la mobilité $\mu_{2}$ dans la région 2 supposée constante en fonction de la température (cf. 2.5.1), nous conduira à poser $\alpha=0$.

2.5.4 Simulation numérique et conclusion. - Dans ce paragraphe nous allons exposer les résultats des calculs, effectués à partir des paramètres de transport élémentaires $\mu_{1}$ (cf. Relation (17)), $n_{1}$ (cf. Relation (20)), $S_{1}$ (cf. Relation (22)), $n_{2}, \mu_{2}$ et $S_{2}$ (cf. Relation (23)); (cf. 2.5.1, 2.5.2, 2.5.3), et donnant les paramètres de transport globaux $\mu_{\mathrm{H}}(11)$ et $S(16)$ pour un échantillon bicouche pris comme référence (cf. 2.1). Cet échantillon, constitué d'une couche principale de Si massif de type $\mathbf{N}$, et d'uné mince couche de Si surdopé $\mathrm{N}^{+}$, aura des paramètres de bases tels que :

- dans la région 1 , le $\mathrm{Si}$ de type $\mathrm{N}$ dopé au phosphore admet les caractéristiques suivantes :

$$
\begin{aligned}
N_{\mathrm{d}} & =10^{17} / \mathrm{cm}^{3} \\
E_{\mathrm{d}} & =-40 \mathrm{meV}[10,12] \\
\mu_{\mathrm{M}} & =1000 \mathrm{~cm}^{2} / \mathrm{V} . \mathrm{s} \\
T_{\mathrm{M}} & =300 \mathrm{~K}
\end{aligned}
$$

- dans la région 2 le $\mathrm{Si}-\mathrm{N}^{+}$dégénéré possède les caractéristiques moyennes :

$$
\begin{aligned}
& n_{2}=10^{19}-5 \times 10^{19} / \mathrm{cm}^{3} \\
& \mu_{2}=10 \mathrm{~cm}^{2} / \mathrm{V} . \mathrm{s} .
\end{aligned}
$$

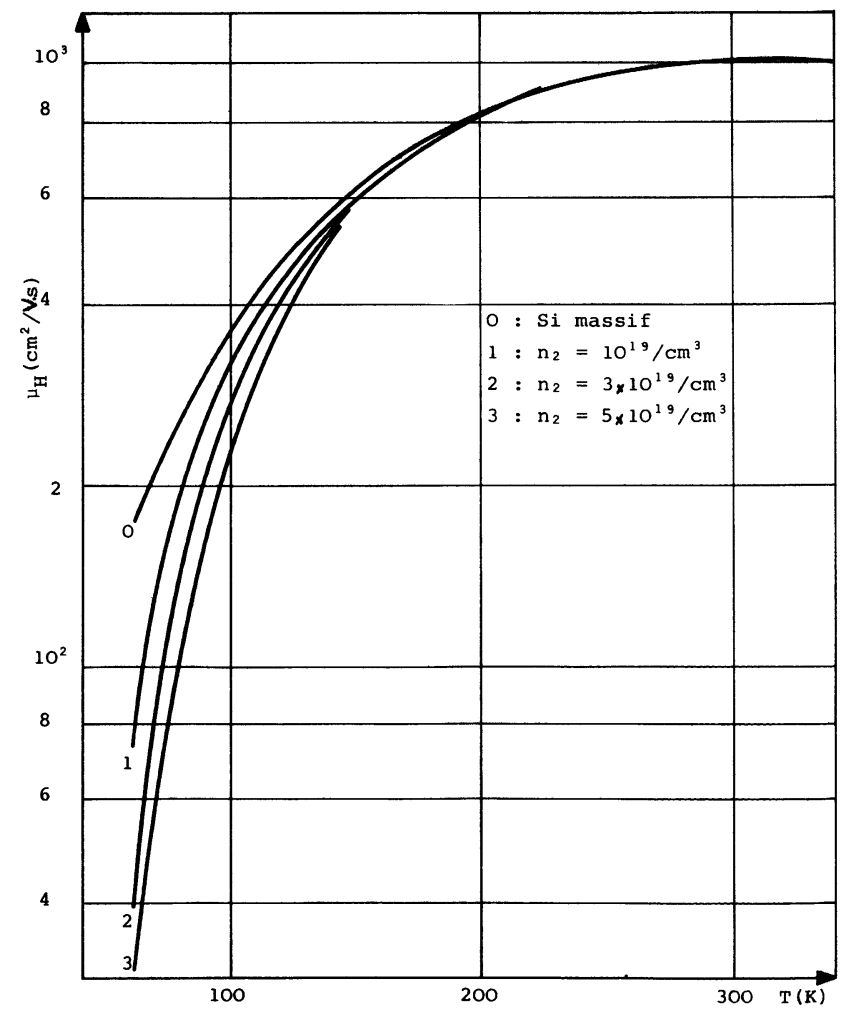

Fig. 2. - Mobilité de Hall en fonction de la température pour l'échantillon bicouche $\mathrm{N}-\mathrm{N}^{+}$et le Si massif.

[Hall mobility vs. temperature for a two-layers $\mathrm{N}-\mathrm{N}^{+} \mathrm{Si}$ sample and for the bulk Si.] 
De plus, nous prendrons une valeur de $\lambda=t_{1} / t_{2}$ telle que nous soyons dans une situation de faible perturbation de la couche principale $\mathrm{N}$, par la couche $\mathrm{N}^{+}$, soit : $\lambda=50$.

Notons que ce dernier paramètre, ainsi que les paramètres des régions 1 et 2 , ont été choisis de façon à être compatibles avec l'analyse ultérieure des échantillons de SSI que nous avons étudiés (cf. $3^{\mathrm{e}}$ partie). En particulier, nous limitons les résultats théoriques à une plage de température comprise entre $60 \mathrm{~K}$ et $360 \mathrm{~K}$ convenable pour notre étude expérimentale.

Sur les figures 2 et 3 sont représentées les variations de la mobilité de Hall et du PTE obtenus à l'aide de l'échantillon de référence précédent $\left(\mathrm{Si}-\mathrm{N}-\mathrm{N}^{+}\right)$. A ce stade, nous constatons que le PTE de notre échantillon transversalement inhomogène est qualitativement très différent de celui du $\mathrm{Si}$ massif $\mathrm{N}$ de même dopage, obtenu en faisant $\lambda$ infiniment grand $\left(t_{2}=0\right)$ (Fig. 3). En effet, à basse température le PTE change de sens de variation par rapport à celui du Si massif $\mathrm{N}$. En revanche, sur la figure 2 , nous constatons que la mobilité de Hall globale de notre échantillon de référence Si-N-N ${ }^{+} n^{\prime} e s t$ pas franchement différente de celle du Si massif $\mathrm{N}$; la différence entre les deux ne s'opérant que sur un plan quantitatif. Même si les écarts relatifs entre les courbes du Si massif $\mathrm{N}$ et de la structure Si-N-N ${ }^{+}$pour l'effet Hall et le PTE sont sensiblement identiques, néanmoins les variations $d u$ PTE portent la marque de linhomogénéité transversale (ici la couche $\mathrm{N}^{+}$) d'une façon beaucoup plus nette que pour celles de la mobilité de Hall, dans les conditions que nous avons considérées. Nous pouvons ajouter cependant, que les conclusions que nous venons de formuler, quant à la meilleure sensibilité du PTE

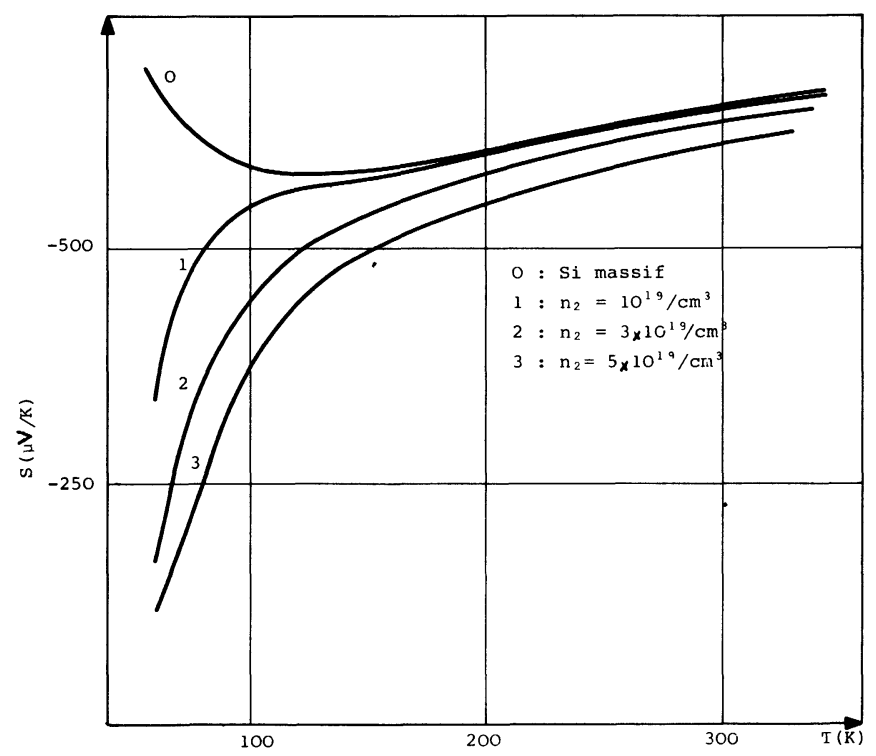

Fig. 3. - Pouvoir thermoélectrique en fonction de la température pour l'échantillon bicouche $\mathrm{N}-\mathrm{N}^{+}$et le $\mathrm{Si}$ massif.

[Thermoelectric power vs. temperature for a two-layers $\mathrm{N}-\mathrm{N}^{+} \mathrm{Si}$ sample and for the bulk $\mathrm{Si}$.] (par rapport à l'effet Hall) vis-à-vis des inhomogénéités transversales, restent valables pour d'autres situations d'inhomogénéités de dopage et de mobilité à condition de maintenir l'hypothèse de dégénérescence dans la couche perturbatrice (ici la couche $\mathrm{N}^{+}$). En d'autres termes cela signifie que l'influence de l'inhomogénéité est notable dans la mesure où elle introduit une différence qualitative dans les propriétés de transport dans l'épaisseur du matériau.

Terminons cette analyse numérique en précisant les sensibilités relatives à chaque paramètre intervenant dans le calcul du PTE $S$ et de la conductivité $\sigma$, et dont nous aurons besoin pour l'ajustement des courbes théoriques aux courbes expérimentales du SSI (3e partie). A cet effet, nous considérons des courbes de référence $\sigma_{0}(T)$ et $S_{0}(\mathrm{~T})$ obtenues pour un jeu de " paramètres de base " $N_{\mathrm{d}}, \mu_{\mathrm{M}}, n_{2}, \mu_{2}, \lambda$ (pour $T_{\mathrm{M}}$ et $E_{\mathrm{d}}$ donnés). Et nous calculerons les écarts relatifs

$$
\begin{array}{ll}
\frac{\Delta S}{S}=\frac{S(T)-S_{0}(T)}{S_{0}(T)}, & \frac{\Delta \sigma}{\sigma}=\frac{\sigma(T)-\sigma_{0}(T)}{\sigma_{0}(T)}, \\
\frac{\Delta S}{S}=\frac{S-S_{0}}{S_{0}}, & \frac{\Delta \sigma}{\sigma}=\frac{\sigma-\sigma_{0}}{\sigma_{0}}
\end{array}
$$

entre ces courbes de référence et les courbes obtenues en modifiant successivement chaque "paramètre de base " ajustable. Nous nous limitons alors à deux températures significatives pour l'ajustement des paramètres : une valeur " basse température " $(T=90 \mathrm{~K})$ et une valeur "haute température" $(T=300 \mathrm{~K})$. Les résultats de ces calculs sont représentés dans le tableau I, et nous permettent de dégager quelques idées générales quant à la sensibilité des paramètres ajustables $N_{\mathrm{d}}, \mu_{\mathrm{M}}, n_{2}, \mu_{2}, \lambda$. Nous pouvons constater que, d'une façon générale, le comportement à " haute température " $(T=300 \mathrm{~K})$ est sensible aux paramètres de la région 1 (couche semiconductrice $\mathrm{Si}-\mathrm{N}$ ), alors que les paramètres de la région 2 (dégénérée $\mathrm{Si}-\mathrm{N}^{+}$) affectent plus particulièrement les valeurs "basses températures » $(T=90 \mathrm{~K})$ de la conductivité et du PTE. C'est ainsi que le plateau du PTE à $300 \mathrm{~K}$ (cf. Fig. 3) est fortement corrélé à la valeur du dopage $N_{\mathrm{d}}$. La valeur à $300 \mathrm{~K}$ de la conductivité dépend principalement du dopage $N_{\mathrm{d}}$ et de la mobilité $\mu_{\mathrm{M}}$. En revanche, les grandeurs de la couche $2\left(N_{2}\right.$ et $\left.\mu_{2}\right)$ sont très influentes sur le PTE et la conductivité à $90 \mathrm{~K}$ et seront par conséquent principalement ajustées aux moyens des données expérimentales « basses températures".

Tout au long de cette partie nous avons montré que les paramètres de transport $\sigma, \mu_{\mathrm{H}}$ et $S$ d'un matériau bicouche, sont notablement modifiés par la présence d'une inhomogénéité transversale. En particulier grâce à l'analyse théorique du début de cette partie, un échantillon de référence constitué d'une couche de $\mathrm{Si}-\mathrm{N}$ et d'une mince couche de $\mathrm{Si}-\mathrm{N}^{+}$, nous a permis de mettre en évidence la supériorité relative du PTE sur leffet Hall vis-à-vis des inhomogénéités de type transversal. C'est la raison pour laquelle, sur un plan expérimental, nous avons porté plus particulièrement notre atten- 
Tableau I. - Sensibilité des valeurs du pouvoir thermoélectrique et de la conductivité aux variations des principaux paramètres ( pour $E_{\mathrm{d}}=-40 \mathrm{meV}$ et $T_{\mathrm{M}}=300 \mathrm{~K}$ ) dans le modèle bicouche.

[Sensitivity of the values of the thermoelectric power and of the conductivity to the variations of the main parameters (for $E_{\mathrm{d}}=-40 \mathrm{meV}$ and $T_{\mathrm{M}}=300 \mathrm{~K}$ ), in the two-layers model.]

\begin{tabular}{|c|c|c|c|c|c|c|c|c|}
\hline & & & & \multicolumn{2}{|c|}{$\Delta S / S$} & \multicolumn{3}{|c|}{$\Delta \sigma / \sigma$} \\
$N_{\mathrm{d}}\left(\mathrm{cm}^{-3}\right)$ & $\mu_{\mathrm{M}}\left(\mathrm{cm}^{2} / \mathrm{V} . \mathrm{s}\right)$ & $n_{2}\left(\mathrm{~cm}^{-3}\right)$ & $\mu_{2}\left(\mathrm{~cm}^{2} / \mathrm{V} . \mathrm{s}\right)$ & $\lambda=\frac{t_{1}}{t_{2}}$ & $T=300 \mathrm{~K}$ & $T=90 \mathrm{~K}$ & $T=300 \mathrm{~K}$ & $T=90 \mathrm{~K}$ \\
\hline $10^{17}$ & 800 & $10^{19}$ & 8 & 40 & \multicolumn{3}{|c|}{ paramètres de référence (-) } \\
\hline $2 \times 10^{17}$ & - & - & - & - & $-9 \%$ & $-1,5 \%$ & $90 \%$ & $38 \%$ \\
\hline- & 600 & - & - & - & $\simeq 0$ & $4 \%$ & $-24 \%$ & $-19 \%$ \\
\hline- & - & $2 \times 10^{19}$ & - & - & $-2 \%$ & $15 \%$ & $+3 \%$ & $22 \%$ \\
\hline- & - & - & 4 & - & $\simeq 0$ & $7 \%$ & $-1,5 \%$ & $-10 \%$ \\
\hline- & - & - & - & 30 & $\simeq 0$ & $-4 \%$ & $\simeq 0$ & $7 \%$ \\
\hline- & - & - & - & 50 & 0 & $2,5 \%$ & 0 & $-3,5 \%$ \\
\hline
\end{tabular}

tion sur l'étude du pouvoir thermoélectrique du SSI, dans lequel la présence d'inhomogénéités transversales est bien établie et donne lieu à une modification notable des propriétés de transport $[6,7,8,30]$.

3. Analyse des résultats expérimentaux du SSI. Dans cette partie après une présentation des résultats expérimentaux concernant le PTE et la conductivité du SSI, nous procéderons à une analyse quantitative du rôle des inhomogénéités transversales sur les propriétés de transport. Cette analyse sera basée sur le modèle développé dans la $2^{\mathrm{e}}$ partie et permettra la comparaison du SSI, à l'échantillon bicouche de référence de structure $\mathrm{Si}-\mathrm{N}-\mathrm{N}^{+}$.

3.1 ECHANTILLONS ET MONTAGE EXPÉRIMENTAL. Les échantillons de SSI que nous avons utilisés ont été réalisés au LETI-MEA du C.E.N. de Grenoble par croissance en phase vapeur (hétéroépitaxie par pyrolyse du silane à $1100^{\circ} \mathrm{C}$ ) d'une couche de silicium sur un substrat de corrindon $\left((001) \mathrm{Si} /(\overline{1} 012) \mathrm{Al}_{2} \mathrm{O}_{3}\right)$. Les films épitaxiés de $\mathrm{Si}$, de $0,7 \mu \mathrm{m}$ d'épaisseur, ont été dopés par implantation ionique de phosphore à des taux compris entre $10^{12} / \mathrm{cm}^{2}$ et de $5 \times 10^{13} / \mathrm{cm}^{2}$. Des contacts ohmiques ont été réalisés par adjonction à chaque extrémité d'une zone $\mathrm{n}^{+}$et d'une métallisation d'aluminium (cf. encastré Fig. 4). Des mesures de PTE et de conductivité électrique ont été réalisées sur ces échantillons, entre $77 \mathrm{~K}$ et $360 \mathrm{~K}$ à l'aide d'un cryostat à conduction solide. Pour la mesure du PTE, nous avons utilisé une méthode dynamique précédemment décrite dans la littérature $[18,19]$. Cette méthode consiste à soumettre l'échantillon à un gradient de température dynamique et à tracer la caractéristique tension Seebeck $\Delta V_{\mathrm{s}}$-différence de température $\Delta T$ (mesuré par thermocouple) pour chaque température entre $77 \mathrm{~K}$ et $360 \mathrm{~K}$. Le PTE est alors déduit par la pente de la caractéristique $\Delta V_{\mathrm{s}}-\Delta T$, dont la linéarité pour des $\Delta T$ faibles garantit l'absence d'hystérésis de réponse échantillon-thermocouple.

Pour la mesure de la conductivité électrique, nous employons une méthode classique à deux fils, suffisante dans notre cas. La résistance électrique des échantillons utilisés est alors mesurée directement sans difficulté due aux contacts vu leur ohmicité.

3.2 Résultats EXPérimentauX. - Les courbes expérimentales donnant le PTE et la conductivité en fonction de la température sont représentées sur les figures 4 et 5 .

On remarquera que, conformément au type de dopage, ici N, le PTE est toujours négatif sur toute la gamme de température explorée. De plus à la température ambiante le PTE prend des valeurs compatibles avec le dopage moyen des échantillons (entre $10^{16}$ et $7 \times 10^{17} / \mathrm{cm}^{3}$ ). En revanche à basse température les variations du PTE, ne sont pas celles attendues pour un semiconducteur (cf. § 2.5.4) et elles ont un comportement inhabituel pour le silicium [19, 29, 35]. Notre analyse qualitative dans un premier temps [20] nous a conduit à penser que la couche de transition $\mathrm{Si}-\mathrm{Al}_{2} \mathrm{O}_{3}$ était vraisemblablement responsable du comportement anormal du PTE à basse température. A cet effet, sachant que l'interface supérieur $\mathrm{Si}_{-} \mathrm{SiO}_{2}$ (la couche de silice d'une vingtaine d'angströms au plus, est de croissance naturelle) pouvait jouer un rôle au même titre que l'interface $\mathrm{Si}-\mathrm{Al}_{2} \mathrm{O}_{3}[5,21]$ nous avons étudié le PTE d'un échantillon ( ${ }^{\circ}$ 11bis sur la Fig. 4) sur lequel une couche d'or a été déposée. La structure en question présentant une structure 


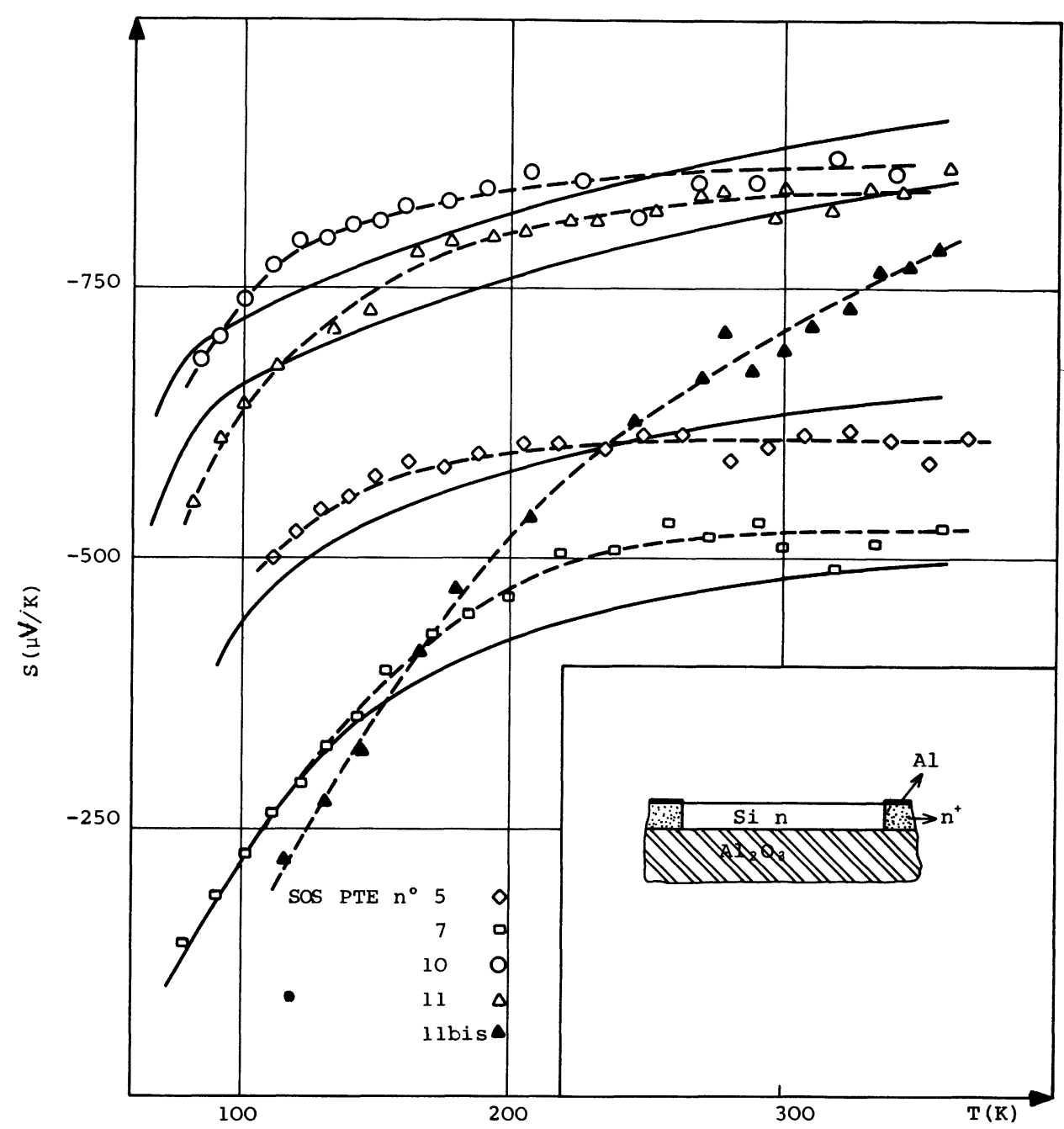

Fig. 4. - Pouvoir thermoélectrique en fonction de la température pour différents échantillons de SSI (les traits pleins correspondent au meilleur ajustement par le modèle bicouche)(voir texte).

[Thermoelectric power vs. temperature for different SOS samples (full lines correspond to the best theoretical fitting by the two-layers model) (see text).]

Schottky Au-Si en surface [17] permet donc, grâce à l'effet de champ ainsi obtenu, vu les différences de travaux de sortie entre $\mathrm{Au}$ et $\mathrm{Si}$, de réduire la contribution sur le PTE, de l'interface supérieur $\mathrm{Si}_{-} \mathrm{SiO}_{2}$. L'effet de la désertion de surface tend ainsi au contraire à accroître l'influence de l'interface inférieure $\mathrm{Si}-\mathrm{Al}_{2} \mathrm{O}_{3}$ et permet donc d'accroître le caractère inhomogène du film de SSI dans la direction épitaxiale. Ce résultat, malgré son caractère qualitatif, confirme que la dégradation du transport dans l'épaisseur du film est imputable à la couche de transition $\mathrm{Si}-\mathrm{Al}_{2} \mathrm{O}_{3}$ et non à l'interface supérieur $\mathrm{Si}_{-} \mathrm{SiO}_{2}$ [30] .

Sur la figure 5 sont représentées les courbes donnant la conductivité $\sigma$ pour les échantillons considérés. La conductivité passe par un maximum pour des températures comprises entre $240 \mathrm{~K}$ et $300 \mathrm{~K}$. Pour des températures supérieures à celle du maximum, la conductivité diminue légèrement laissant présager une limitation de la mobilité par la diffusion du réseau (phonons acoustiques). En dessous du maximum, quand la température s'abaisse, la conductivité diminue fortement (d'un facteur 3 à 4), ce qui est en accord avec une diminution du nombre de porteurs majoritaires par désactivation thermique du semiconducteur.

3.3 InTERPRÉTATION ET DISCUSSION. - L'objet de ce paragraphe est d'exploiter l'analyse quantitative que nous avons présentée dans la $2^{\mathbf{e}}$ partie, pour l'interprétation des résultats expérimentaux concernant le SSI étudié. Comme nous l'avons montré antérieurement $[20,22]$ un modèle bicouche tel celui décrit dans la partie précédente, est susceptible de rendre compte des variations du PTE et de la conductivité du SSI en fonction de la température. Nous considérons, dans ce cas, que le SSI étudié est un matériau suffisamment inhomogène transversalement pour être assimilé à un matériau constitué de deux régions très nettement différentes sur le plan du transport électrique; la partie supérieure du film semblable au $\mathrm{Si}$ massif et la couche de transition à 


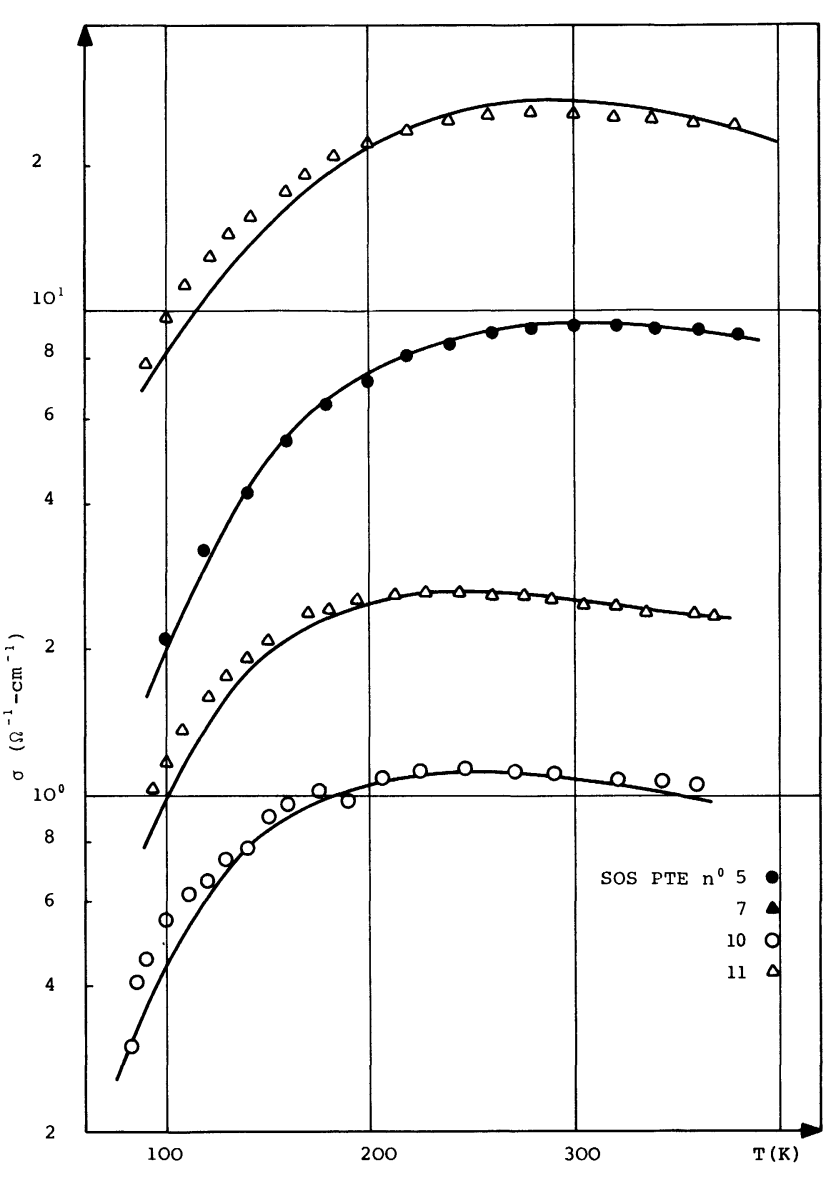

Fig. 5. - Conductivité en fonction de la température pour différents échantillons de SSI (les traits pleins correspondent au meilleur ajustement théorique par le modèle bicouche) (voir texte).

[Conductivity vs. temperature for different SOS samples (full lines correspond to the best theoretical fitting by the two-layers model) (see text).]

l'interface $\mathrm{Al}_{2} \mathrm{O}_{3}$-Si de nature dégénérée. $\mathrm{A}$ l'aide du modèle théorique précédent $(\S 2.5)$, nous avons alors procédé à l'ajustement des courbes théoriques donnant le PTE et la conductivité en fonction de la tempéra- ture sur les points expérimentaux du PTE et de la conductivité pour les différents échantillons de SSI étudiés. Sur les figures 4 et 5 sont représentées les courbes théoriques correspondant au meilleur ajustement. Nous pouvons, par conséquent, dire qu'en première approximation, le modèle physique considéré rend compte d'une manière satisfaisante des propriétés de transport dans le SSI étudié dans la gamme de température explorée.

Nous avons reporté dans le tableau II, les valeurs correspondantes des 5 paramètres indépendants déduits de l'ajustement. Dans celui-ci sont donnés les paramètres utilisés dans la $2^{\mathrm{e}}$ partie tels que le dopage $N_{\mathrm{d}}$, la mobilité $\mu_{\mathrm{M}}$ de la couche principale du film ainsi que la densité de porteurs $n_{2}$, la mobilité $\mu_{2}$ de la couche de transition dont l'épaisseur est déduite de la valeur de $\lambda\left(\lambda=t_{1} / t_{2}\right.$, cf. Fig. 1). Pour que l'analyse numérique issue de l'ajustement soit complète, la précision de chaque paramètre doit être connue. A cet effet, nous comparons les écarts relatifs existant entre les courbes théoriques et les courbes expérimentales, aux écarts relatifs obtenus lors du test de sensibilité des paramètres présenté dans le tableau I (cf. § 2.5.4). Compte tenu de ce principe, une estimation supérieure de la précision sur chaque paramètre peut être déduite. C'est ainsi que la densité des donneurs $N_{\mathrm{d}}$ est fiable à un facteur 2 près et la mobilité maximum $\mu_{\mathrm{M}}$ ne présente pas plus de $10 \%$ d'erreur pour la couche supérieure du film. De même pour la couche de transition la densité de porteur $n_{2}$ s'obtient à un facteur 2 près. Quant au rapport géométrique $\lambda$, exprimant l'importance relative de la couche $\mathrm{N}$, sur la couche $\mathrm{N}^{+}$, il est déduit à environ $30 \%$ près.

Les résultats du tableau II montrent que le SSI étudié est un matériau franchement inhomogène dans la direction épitaxiale. La partie supérieure du film présente un caractère semiconducteur analogue au $\mathrm{Si}$ massif. Cependant, on constate une dégradation notable de la mobilité de l'ordre de $50 \%$ par rapport à celle du Si massif $[14,29]$. Il n'existe pas dans la littérature une cohérence dans l'explication de cette

Tableau II. - Résultats de l'ajustement précisant la valeur des 5 paramètres indépendants $\left(N_{\mathrm{d}}, \mu_{\mathrm{M}}, n_{2}, \mu_{2}, \lambda\right)$ pour différents échantillons de SSI d'après le modèle bicouche (la valeur $E_{\mathrm{d}}$ de l'énergie d'ionisation du phosphore a été considérée connue; celles de la température $T_{\mathrm{M}}$ du maximum de la conductivité sont issues des mesures).

[Fitting results giving the value of the 5 independent parameters $\left(N_{\mathrm{d}}, \mu_{\mathrm{M}}, n_{2}, \mu_{2}, \lambda\right)$ for different SOS samples according the two-layers model (the value $E_{\mathrm{d}}$ of the phosphorous impurity ionization energy is supposed known; the values of the temperature $T_{\mathrm{M}}$ corresponding to the maximum of conductivity are deduced from experimental curves).]

\begin{tabular}{|c|c|c|c|c|c|c|c|c|}
\hline $\begin{array}{c}\text { Echantillon } \\
n^{\mathrm{o}}\end{array}$ & $E_{\mathrm{d}}(\mathrm{meV})$ & $N_{\mathrm{d}}\left(\mathrm{cm}^{-3}\right)$ & $\mu_{\mathrm{M}}\left(\mathrm{cm}^{2} / \mathrm{V} . \mathrm{s}\right)$ & $T_{M}(\mathrm{~K})$ & $\mu_{2}\left(\mathrm{~cm}^{2} / \mathrm{V} . \mathrm{s}\right)$ & $\lambda=\frac{t_{1}}{t_{2}}$ & $n_{2}\left(\mathrm{~cm}^{-3}\right)$ & $t_{2}(\AA)$ \\
\hline$\underline{n^{0}}$ & - & - & - & - & - & - & - & - \\
\hline 7 & -40 & $3 \times 10^{17}$ & 500 & 260 & 8 & 50 & $2 \times 10^{20}$ & 140 \\
\hline 5 & - & $1 \times 10^{17}$ & 550 & 290 & 2 & 50 & $8 \times 10^{19}$ & 140 \\
\hline 10 & - & $7 \times 10^{15}$ & 1000 & 250 & 2 & 70 & $1 \times 10^{19}$ & 100 \\
\hline 11 & - & $1 \times 10^{16}$ & 1100 & 250 & 3 & 50 & $1 \times 10^{19}$ & 140 \\
\hline
\end{tabular}


dégradation. On peut seulement signaler quelques éléments de réponses qui abondent dans ce sens.

En plus des mécanismes de diffusion communément admis (phonons acoustiques et impuretés ionisées [7, 8]), il faut également citer les mécanismes de diffusion sur les impuretés neutres $[5,7]$ ou bien encore sur des défauts cristallographiques (dislocations, joints de grains [5]). Il est évident que, dans ce cas, les lois des mobilités en fonction de la température doivent être modifiées mais malheureusement la littérature à cet égard n'est pas unanime sur le type de loi utilisable [10, $12,13]$. Une autre raison de la diminution de la mobilité dans le SSI de type $\mathrm{N}$ est la présence de contraintes énormes (de l'ordre de $7 \mathrm{kbar}$ [25]) dont le film est le siège. La cause principale de ces contraintes provient de la dilatation différentielle entre le $\mathrm{Si}$ et le saphir, résultant de la relaxation thermique inhérente au processus d'épitaxie par pyrolyse du silane [5, 25]. La théorie de l'élastorésistance [26] prévoit à cet effet un abaissement de l'ordre de $30 \%$ de la mobilité de dérive. Néanmoins seul ce phénomène ne peut entièrement expliquer la dégradation de la mobilité dans la partie supérieure du film [35]. La forte densité de défauts neutres ou ionisés, croissante vers l'interface $\mathrm{Si}-\mathrm{Al}_{2} \mathrm{O}_{3}[21,30,31]$ a également pour conséquence de diminuer la valeur de la mobilité. De plus, il est à noter que la diffusion sur des centres " neutres", n'altèrent pas sensiblement, les variations relatives de la mobilité avec la température $[10,12,13]$. C'est la raison pour laquelle les lois de mobilités en $T^{-3 / 2}$ (phonons acoustiques) et en $T^{3 / 2}$ (impuretés ionisées) pour la partie supérieure du film, sont grossièrement vérifiées [7, 8, 30].

En ce qui concerne la couche de transition, nos résultats montrent que celle-ci présente une forte densité de porteurs, en accord qualitatif avec ceux d'autres auteurs $[8,23,30]$. De plus, les valeurs trouvées échelonnées entre $10^{19}$ et $10^{20} / \mathrm{cm}^{3}$ sont a posteriori compatibles avec l'hypothèse de dégénérescence requise par le modèle physique utilisé $[14,29]$. La mobilité $\mu_{2}$ de cette couche est très nettement inférieure à la mobilité de la couche supérieure (de l'ordre de 100 fois environ). Ce résultat, qui, en lui-même, ne constitue pas une surprise puisqu'il a été signalé par ailleurs $[8,9,30]$, confirme la dégradation du transport vers l'interface $\mathrm{Si}-\mathrm{Al}_{2} \mathrm{O}_{3}$. A cet égard, cela signifie que la densité de "défauts " ou plus généralement de centres diffuseurs va croissante vers l'interface $\mathrm{Si}-\mathrm{Al}_{2} \mathrm{O}_{3}[5,21,30,31]$. L'épaisseur de la couche de transition déduite dans le tableau II, est de l'ordre de 100 à $150 \AA$; cette valeur, en accord avec celles des références $[3,8,23]$ montre que la couche de transition dans le SSI étudié est localisée sur une faible épaisseur du film à l'interface $\mathrm{Si}-\mathrm{Al}_{2} \mathrm{O}_{3}$. A cet égard, cela conforte notre hypothèse de travail dans le modèle physique de la $2^{\mathrm{e}}$ partie, selon laquelle, cette couche constituant l'inhomogénéité transversale, peut être considérée comme une perturbation vis-à-vis de la couche supérieure du film. Notons que l'origine physique de cette couche de transition dans le SSI est très controversée. Heiman [23] estime que celle-ci est une couche d'accumulation imputable à une forte densité de charges fixes à l'interface $\mathrm{Si}_{-} \mathrm{Al}_{2} \mathrm{O}_{3}$ [24]. En revanche, Elliot et Anderson [8], pensent qu'elle résulte d'un accroissement de la densité de donneurs, pour lesquels l'interface $\mathrm{Si}-\mathrm{Al}_{2} \mathrm{O}_{3}$ agirait comme un « puit » d'impuretés.

Dans notre cas, compte tenu de l'épaisseur trouvée (100 à $150 \AA$ ) cela correspond à une densité de charges de l'ordre de $10^{13} / \mathrm{cm}^{2}$ compatible avec la plage donnée par Heiman [23]. Nous pensons, pour notre part, que l'hypothèse de la couche d'accumulation semble plausible dans la mesure où les épaisseurs trouvées (100-150 $\AA$ ) sont cohérentes avec les épaisseurs admises pour une couche d'accumulation dégénérée [33].

Cependant, l'origine physique de cette couche de transition n'est nullement comprise dans sa plénitude et certainement d'autres analyses expliquant la dégénérescence locale du matériau sont envisageables. Nous citerons, par exemple, les effets du désordre à courte et longue portée sur le silicium épitaxié à l'interface $\mathrm{Si}-\mathrm{Al}_{2} \mathrm{O}_{3}$, à l'image de ce qu'il advient à l'interface $\mathrm{Si}_{-} \mathrm{SiO}_{2}$ [27], ou bien encore l'effet des fluctuations de potentiel électrique entraînant la formation de queue de bande d'états localisés [34] dont le rôle exact sur les propriétés de transport reste encore à déterminer.

4. Conclusion. - Le travail que nous venons de présenter met en valeur l'utilisation des mesures de pouvoir thermoélectrique pour l'analyse des propriétés de transport des semiconducteurs inhomogènes.

Plus particulièrement, nous avons démontré que pour des matériaux «bicouches », tels ceux provenant des épitaxies, le pouvoir thermoélectrique est beaucoup plus sensible que l'effet Hall, traditionnellement employé.

Evidemment, l'exploitation parallèle des mesures de conductivité électrique, d'effet Hall et de pouvoir thermoélectrique en fonction de la température, cernerait mieux les paramètres du matériau étudié.

Nous avons appliqué nos analyses théoriques dans le cas du Silicium sur Saphir de type $N$ et de structure manifestement bicouche $\left(\mathrm{N}-\mathrm{N}^{+}\right)$. Ainsi nous avons étudié parallèlement la conductivité électrique et le pouvoir thermoélectrique du matériau. Nos résultats expérimentaux et leur précision rend, dans ce cas de matériau franchement bicouche, l'utilisation de l'effet Hall redondante.

Les mobilités et les densités des porteurs ainsi que leur évolution avec la température ont été déduites.

Nous confirmons que le matériau est composé d'une couche monocristalline principale et d'une couche de transition de type dégénéré d'une épaisseur de l'ordre de $150 \AA$. Les mesures que nous avons présentées ne permettent pas de se prononcer sur la nature de la couche de transition (région surdopée 
ou couche d'accumulation), nous traiterons ce problème dans un prochain article grâce à une analyse plus affinée des mesures du pouvoir thermoélectrique. Ce travail est actuellement en cours [35].
Remerciements. - Ce travail a pu être réalisé grâce au financement du Groupement des Circuits Intégrés au Silicium (GCIS) du CNRS.

\section{Bibliographie}

[1] Borel, J., L'onde électrique 58 (1978) 812.

[2] Preuss, E., et Schlotterer, H., Solid State Devices Conf. Series n ${ }^{\circ} 40$, Institute of Physics, Ed. London (1977) 7-31.

[3] Cristoloveanu, S., Chovet, A., Kamarinos, G., Solid State Electron. 21 (1978) 1563.

[4] Kranzer, D., Appl. Phys. Lett. 25, 2 (1974) 103.

[5] Cullen, G. W. and WANG, C. C., Heteroepitaxial semiconductors for Electronic Devices (Springer Verlag, New York) 1978.

[6] Lee, J. H. and Cristoloveanu, S., 8th Inter. Vacuum Congress, Cannes, France, Vol. I (1980) proceed. p. 550.

[7] IPRI, A. C., J. Appl. Phys. 43 (1972) 2770.

[8] Elliot, A., Anderson, J., Solid State Electron. 15 (1972) 531.

[9] Gentil, P., Electron. Lett. 16 (1980) 617.

[10] Vapallle, A., Physique des dispositifs à semiconducteurs - Electronique du silicium homogène (Masson et Cie, Paris) 1970.

[11] Cristoloveanu, S., Lee, J. H. et Chovet, A., Revue Phys. Appl. 15 (1980) 725.

[12] Sмiтн, R. A., Semiconductors (Cambridge, University Press) 1978.

[13] NAG, B. R., Electron transport in Semiconductor compounds (Springer Verlag, Berlin) 1980.

[14] Morin, F. and Maita, J., Phys. Rev. 96 (1955) 28.

[15] Herring, C., Phys. Rev. 96 (1954) 1163.

[16] Mac Donald, D. K., Thermoelectricity : An introduction to the principles (Wiley, New York) 1962.

[17] Sze, S. M., Physics of Semiconductor devices (Wiley, New-York) 1965.

[18] Chaikin, P. and Fwak, J., Rev. Sci. Instrum. 46 (1975) 2.

[19] Ghibaudo, G. et Stioui, C., Rapport DEA (41 pages) USM Grenoble (1979) non publié.
[20] Ghibaudo, G., Hsia, C. Y., Kamarinos, G.

(a) ESSDERC 80, York (England), Vol. 4HEPS (1980) 143 ;

(b) IEEE SOS Workshop, Cupertino (USA) (1980).

[21] Mercier, J., J. Electrochem. Soc. 118 (1971) 962.

[22] Ghibaudo, G., Hsia, C. Y., Kamarinos, G., 8th Inter. Vacuum Congress, Cannes, France, Vol. I (1980) 459.

[23] Heiman, F. P., Appl. Phys. Lett. 11 (1967) 4.

[24] Gentil, P. and Chausse, S., Solid State Electron. 20 (1977) 935.

[25] Englert, T., Abstreiter, G. and Pontcharra, J., Solid State Electron 23 (1980) 31.

[26] Hynicek, J., J. Appl. Phys. 45 (1974) 6.

[27] Motr, N., Davis, E., Electronic Processes in non crystalline materials (Clarendon Press, London) 1979.

[28] Putley, E. H., The Hall Effect and semiconductors physics (Dover Publications Inc., New-York) 1960.

[29] Wolf, J., Silicon Semiconductor Data (Pergamon Press, London) 1969.

[30] Nishi, Y. and HaRA, H., Jap. J. Appl. Phys. Supp. 27 (1978) 27.

[31] Trilhe, J., Blanchard, B. and Borel, J., 3rd Int. Conf. on Solid Surfaces, Vienne (Autria) (1977) 541.

[32] Englert, T., Landwehr, G., Pontcharra, J. and Dorda, G., Surf. Sci. 98 (1980) 427.

[33] Many, A., Goldstein, Y., Grover, N., Semiconductor surfaces (North Holland, Amsterdam) 1965.

[34] Robert, J. L., Pistoulet, B., Raymond, A. and Dusseau, J. M., J. Appl. Phys. 50 (1979) 349.

[35] Ghibaudo, G., Thèse INP Grenoble (1981). 